\title{
Mars: Mapping surface units by means of statistical analysis of TES spectra
}

\author{
F. Altieri ${ }^{1}$ and G. Bellucci ${ }^{1}$
}

\begin{abstract}
CNR, Istituto di Fisica dello Spazio Interplanetario 00133 Rome, Italy
Received 28 November 2001 / Accepted 3 December 2002

Abstract. Thermal Emission Spectrometer (TES) data from the Mars Global Surveyor (MGS) mapping phase have been processed to identify regions with unique spectral features for new clues on the Martian surface composition. For this purpose we have developed a procedure to search and map band absorptions related to presence of different surface minerals on a spatial scale of a few kilometers. Data used in this study cover the March 1999-July 2000 period, corresponding to $120^{\circ}<L_{\mathrm{s}}<250^{\circ}$ on Nili Fossae, Sinus Meridiani and Valles Marineris regions, where outcrops of olivines and hematite have been identified in previous studies. We have tested the validity of our procedure on these areas and then extended our analysis to other portions of the planet. The data have been assembled in $10^{\circ} \times 10^{\circ}$ emissivity spectra cubes with $16 \times 16$ pixels per square degree. The Principal Components Analysis (PCA) has been used to identify spectra with very low contamination by atmospheric aerosols. It has been applied in two spectral ranges: $300-550 \mathrm{~cm}^{-1}(\sim 18.2-33.3 \mu \mathrm{m})$ and $815-1143 \mathrm{~cm}^{-1}(\sim 8.7-12.3 \mu \mathrm{m})$. By means of PCA we have selected three principal spectral classes: spectra with a high content of dust, spectra with a high content of water-ice and spectra with a lower contamination by dust and water-ice. To identify emission spectra with interesting features likely related to surface minerals we have selected, in the last class, data with high spectral variance between $300-550 \mathrm{~cm}^{-1}$, the range where the hematite and olivine bands have been found.
\end{abstract}

Key words. planets and satellites: individual: Mars - techniques: spectrometric - methods: data analysis

\section{Introduction}

Over the last few years numerous attempts have been made to determine the Martian surface and atmosphere composition to understand the past geologic processes and past climates of the planet. A large amount of data has been collected thanks to the instruments aboard Mariner 6-7-9, Viking 1-2, and Phobos 2. Since September 1997, the Mars Global Surveyor (MGS) NASA mission has been orbiting around Mars and the Thermal Emission Spectrometer (TES) has collected an enormous amount of data. The TES objectives are (1) to determine and map the composition of surface minerals, rocks and ices; (2) to study the composition, particle size and spatial and temporal distribution of atmospheric dust; (3) to locate water-ice and $\mathrm{CO}_{2}$ condensate clouds and determine their temperature, height and condensate abundance; (4) to study the growth, retreat and total energy balance of the polar cap deposits; and (5) to measure the thermophysical proprieties of Martian surface materials (Christensen et al. 1992). The MGS spacecraft mapping phase began in March 1999 after the completion of the aerobraking period. To date TES has acquired spectra over an entire Martian year in a circular polar orbit with equator crossing at $2 \mathrm{H}$ and $14 \mathrm{H}$ Mars local time (24 $\mathrm{H}$ equals one Martian day). The interpretation of this huge

Send offprint requests to: F. Altieri,

e-mail: francesca.altieri@isfi.rm.cnr.it quantity of data is complicated by the difficulty of discriminating the different contributions of surface and atmospheric components. One of the principal scientific objective of the TES investigation is to determine the composition of Martian surface. Included in its spectral range are features caused by atmospheric components: $\mathrm{CO}_{2}$ and water vapour gases, dust and water ices aerosols. The measured spectrum is the result of emission, absorption and transmission of energy by surface materials and by atmospheric components. The surficial dust layer has a spectral masking effect, generally smoothing the soil band contrast (Gaff et al. 2001; Crisp \& Bartholomew 1992). The main $\mathrm{CO}_{2}$ gas absorption is centered at $667 \mathrm{~cm}^{-1}$ $(15 \mu \mathrm{m})$; other weaker $\mathrm{CO}_{2}$ absorptions are centered at 550, 790, 961, 1064, 1260 and $1366 \mathrm{~cm}^{-1}$ (Maguire 1977). Dust aerosols have a principal broad band centered at $1075 \mathrm{~cm}^{-1}$ and a minor broad band centered at $480 \mathrm{~cm}^{-1}$ (Huguenin 1987; Grassi \& Formisano 2000). Water-ice aerosols have a broad peak near $825 \mathrm{~cm}^{-1}$, a sharper peak at $229 \mathrm{~cm}^{-1}$ and minor absorption at wavenumbers $>1000 \mathrm{~cm}^{-1}$, water vapour has absorption at $200-400 \mathrm{~cm}^{-1}$ and $1400-1800 \mathrm{~cm}^{-1}$ (Smith et al. 1999).

In this preliminary work we have developed a rapid method to distinguish spectra strongly contaminated by atmospheric aerosols (dust and ices), and to identify interesting surface spectral features in one single TES detector spectrum. The method is applicable to imaging spectrometry data gathered by 
future space instrumentation as well. In Sect. 2 we describe briefly the TES instrument characteristics, in Sect. 3 we describe the data used in our study, in Sect. 4 we show the statistical method we have used to select the spectra reported in Sect. 5 and, finally, in Sect. 6 we draw some conclusions.

\section{TES instrument}

The Mars Global Surveyor Thermal Emission Spectrometer consists of a Michelson interferometric spectrometer collecting thermal infrared spectra between 6 and $50 \mu \mathrm{m}(200-$ $\left.1650 \mathrm{~cm}^{-1}\right)$, with additional broadband visible $(0.3-2.7 \mu \mathrm{m})$ and thermal $(5.5-100 \mu \mathrm{m})$ channels (Christensen et al. 1992). Six detectors in a three-by-two array simultaneously take infrared spectra with a selectable sampling of 5 or $10 \mathrm{~cm}^{-1}$ containing, respectively, 246 or 143 spectral channels. The instantaneous field of view (IFOV) of each detector is $\sim 8.5 \mathrm{mrad}$ corresponding to a spatial resolution of $\sim 3 \mathrm{~km}$ in the final MGS mapping orbit altitude of $\sim 380 \mathrm{~km}$. A pointing mirror allows TES to carry out periodic view of an aboard calibration blackbody and cold space, as well as measures in the atmosphere above the limb. The TES noise equivalent spectral radiance is $\sim 2.5 \times 10^{-8} \mathrm{~W} \mathrm{~cm}^{-2} \mathrm{sr}^{-1} / \mathrm{cm}^{-1}$ from 300 to $1400 \mathrm{~cm}^{-1}$, increasing to $\sim 6 \times 10^{-8} \mathrm{~W} \mathrm{~cm}^{-2} \mathrm{sr}^{-1} / \mathrm{cm}^{-1}$ at $250 \mathrm{~cm}^{-1}$ and $\sim 6 \times$ $10^{-8} \mathrm{~W} \mathrm{~cm}^{-2} \mathrm{sr}^{-1} / \mathrm{cm}^{-1}$ at $1650 \mathrm{~cm}^{-1}$. Absolute radiometric accuracy has been determined from pre-launch data to better than $\sim 4 \times 10^{-8} \mathrm{~W} \mathrm{~cm}^{-2} \mathrm{sr}^{-1} / \mathrm{cm}^{-1}$ from 300 to $1400 \mathrm{~cm}^{-1}$ (Christensen et al. 1999; Christensen et al. 2000a; Christensen et al. 2001). These random errors correspond to a noise equivalent delta emissivity (NE $\Delta \epsilon)$ of 0.004 at $1000 \mathrm{~cm}^{-1}$ and 0.002 at $400 \mathrm{~cm}^{-1}$ for a surface temperature of $275 \mathrm{~K}$ (Christensen et al. 2001b).

\section{Data set}

Data used in this study cover the period from March 1999 to July 2000, corresponding to an areocentric longitude of the Sun $\left(L_{\mathrm{S}}\right)$ between $120^{\circ}$ and $250^{\circ}$. We have initially selected three regions: the first includes the region of Nili Fossae, where the largest outcrop of olivine has been found (Hoefen et al. 2000; Hoefen \& Clark 2001; Hamilton et al. 2001); the others are on Valles Marineris and Sinus Meridiani, two of the three regions where TES team has identified outcrops of hematite (Christensen et al. 2001a; Lane et al. 2001; Christensen et al. 2000b). Then we have extended our analysis to Sinai Planum, to a region between Syrtis Major and northeastern Arabia Terra and to a portion of $60^{\circ} \times 60^{\circ}$ on the Martian surface between $180^{\circ}$ and $240^{\circ} \mathrm{W}$ and $-30^{\circ}$ and $30^{\circ} \mathrm{N}$. The data were collected during the MGS mapping phase, with a constant orbit and a constant spatial resolution, so that they are ideal to be visualized as cube images, having two spatial dimensions and a third spectral dimension.

The spectral calibrated radiance has been converted to emissivity by (1) deriving the brightness temperature at each wavenumber; (2) assuming that the maximum of the brightness temperature around 1230 and $1300 \mathrm{~cm}^{-1}$ is the Christiansen peak (Christensen \& Harrison 1993; Ruff et al. 1997); (3) setting the maximum brightness temperature equal to the surface kinetic temperature; and (4) computing the emissivity at each wavenumber by dividing the Planck function at the surface kinetic temperature (Christensen et al. 2000b). The assumption that the surface materials have unit emissivity at the point of maximum brightness temperature for the thermal infrared emission has been demonstrated to be valid to within $\sim 3 \%$ for a wide range of minerals, rocks and soils (Salisbury et al. 1991; Ruff et al. 1997).

The emissivity spectra have been assembled in $10^{\circ} \times$ $10^{\circ}$ cubes with $16 \times 16$ pixels per square degree and $143 \mathrm{spec}-$ tral channels. For all cubes we have considered $\sim 17000$ spectra with an emission angle between $\sim 30^{\circ}$ and $60^{\circ}$, corresponding to a Martian local time of $\sim 14.00^{\mathrm{m}}$ and a mean surface temperature of $\sim 280 \mathrm{~K}$. All data have spectral sampling of $10 \mathrm{~cm}^{-1}$ and nadir view. Because our aim was to obtain a good spatial coverage of the examined regions, we have not constrained the data based on dust and water-ice opacities.

\section{Data analysis and results}

Our aim was to find a procedure which allowed us: (1) to identify the main spectral types responsible for the observed spectral variation, and (2) identify regions or single TES pixels having unique spectral contrast in the $300-500 \mathrm{~cm}^{-1}$ spectral range. For this purpose, we have computed the Principal Components Analysis (PCA) and the CHI-square error (CHISQR) of a linear fit on some selected TES spectral channels. Combining these two method of analysis we have been able to isolate spectra with interesting features between 300 and $500 \mathrm{~cm}^{-1}$ spectral domain.

PCA scatter plot. PCA is a technique commonly used in the interpretation of terrestrial and spacecraft multispectral imaging (Siegal \& Gillespie 1980; Ardvison et al. 1982; Bell III et al. 1992). PCA involves the calculation of the eigenvectors of the variance-covariance matrix of data set and then the transformation of data onto a set of orthogonal axes that are linear combinations of the original data (Davis 1986). The first axis, or Band 1, contains the greatest amount of variance, with subsequent components containing decreasing amount of variance. To avoid water vapour and $\mathrm{CO}_{2}$ main features and TES wavenumbers where the noise is higher, we have limited the PCA analysis to some specific spectral channels and selected two main spectral ranges. The first range is between 300 and $550 \mathrm{~cm}^{-1}$ (hereafter Range 1), where the hematite and olivine signatures have been found. For the second range we have chosen spectral channels between 815 and $1143 \mathrm{~cm}^{-1}$ (hereafter Range 2), where the atmospheric aerosols have deepest absorptions. We have found that (1) the PCA first component (Band 1) in the Range 1 is well correlated with the albedo (the correlation between the $20 \mu \mathrm{m}$ spectral channel and the albedo was observed in Viking IRTM data as well (Christensen 1998)); and (2) the use of the second component (Band 2) in the Range 1 and Range 2 to search for spectral variance related to no-dust composition is the most convenient.

The PCA algorithm has been applied to different $10^{\circ} \times$ $10^{\circ}$ emissivity cubes showing similar results. In this section we discuss the case of the Nili Fossae cube, where Hoefen et al. (2000) have identified unique spectral features related 


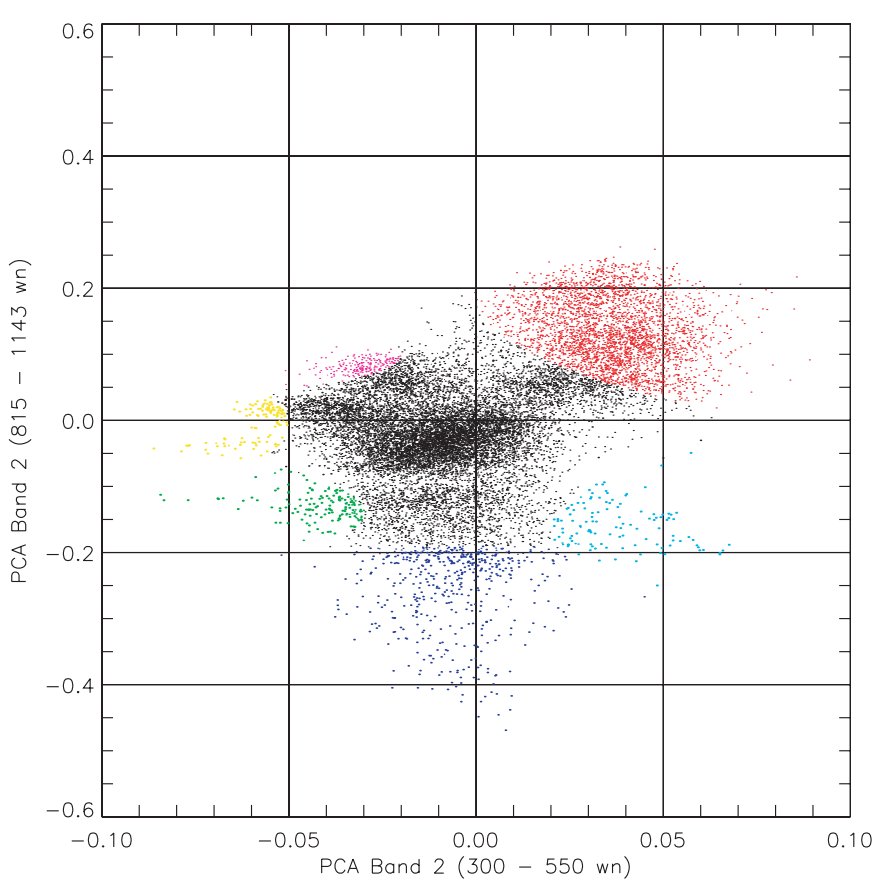

Fig. 1. a) PCA scatter plot of spectra from Nili Fossae region cube.

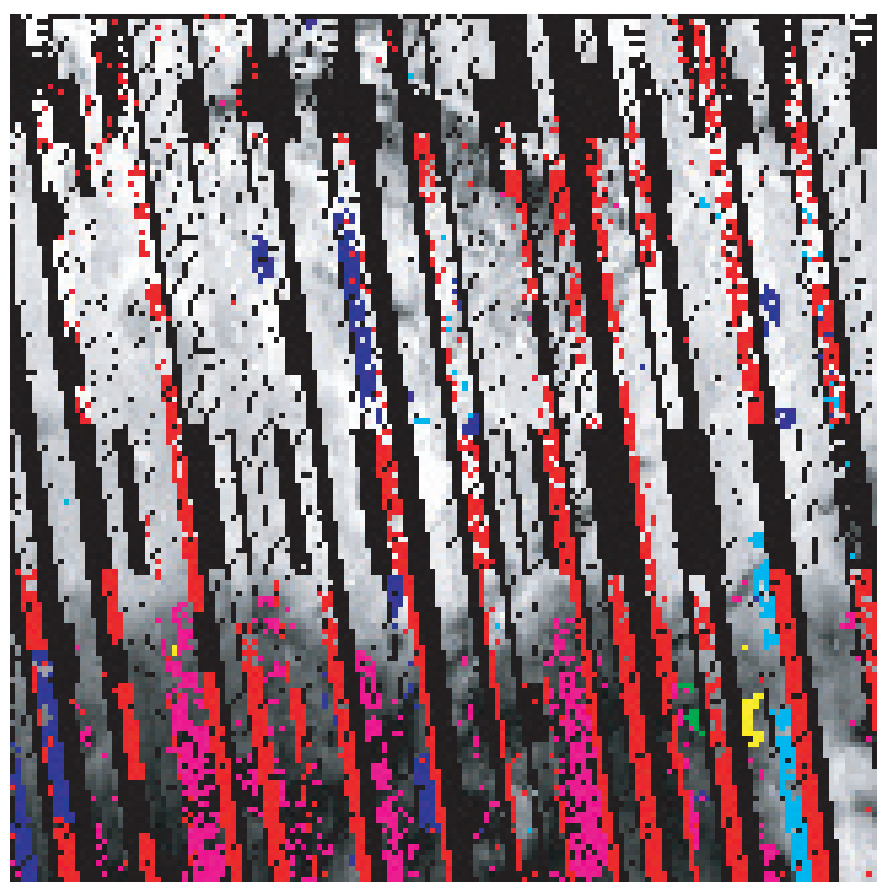

Fig. 1. b) Location of the spectral classes on the albedo map. Spatial coverage $20^{\circ} \mathrm{N}<$ Lat $<30^{\circ} \mathrm{N}$ and $280^{\circ} \mathrm{W}<$ Lon $<290^{\circ} \mathrm{W}$. Black pixels have no TES data in the $L_{\mathrm{s}}$ range used in this study.

to olivine, and the Sinus Meridiani cube, where the largest hematite outcrop has been found (Christensen et al. 2000b).

The south eastern corner of the Nili Fossae cube shows a local maximum in emissivity at 451 and $377 \mathrm{~cm}^{-1}$ and a minimum at $408 \mathrm{~cm}^{-1}$. Using $5 \mathrm{~cm}^{-1} \mathrm{~cm}$ sampling spectra Hoefen et al. (2000) have seen also a maximum at $303 \mathrm{~cm}^{-1}$ and a minimum at $292 \mathrm{~cm}^{-1}$ that are not well identifiable in our data because of lower SNR in this spectral range and water vapour features. They attributed these maxima to Christiansen peaks,

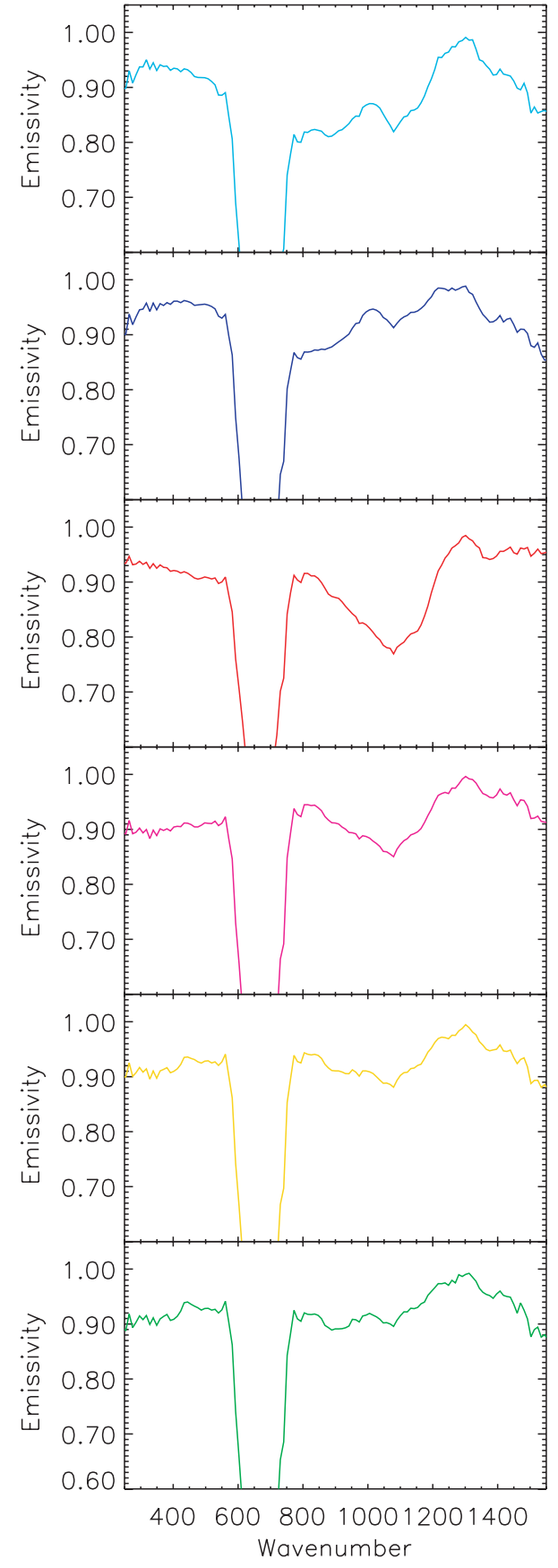

Fig. 1. c) Mean spectra of the classes reported in Fig. 1a. Cyan and blue spectra have typical features of water ice. The red spectrum shows the principal dust band between 850 and $1200 \mathrm{~cm}^{-1}$ and the minor dust band between 300 and $550 \mathrm{~cm}^{-1}$. Violet spectra are spread on albedo map dark regions while yellow and green spectra are well grouped on the map and show the olivine features.

with similarities with sulfides and olivine. Figure 1a shows Range 1 - Range 2 PC2 Scatter Plot of the cube. The spatial coverage is of $20^{\circ} \mathrm{N}<$ Lat $<30^{\circ} \mathrm{N}$ and $280^{\circ} \mathrm{W}<$ Lon $<$ $290^{\circ} \mathrm{W}$. Each point on the scatter plot corresponds to an emissivity spectrum of the cube. The spectral units are identified on the scatter plot using different colors and their position on the $10^{\circ} \times 10^{\circ}$ albedo map is reported below (Fig. 1b). This map 
has been assembled using the TES broadband visible bolometer data. Dark pixels on the map have no data in the $L_{\mathrm{s}}$ period selected for our study. Mean spectra of different spectral units are shown in Fig. 1c. Cyan and blue spectra have typical features of water ice, they differ in the dust content, higher in the cyan spectrum (Christensen et al. 2000b), and their locations on the scatter plot is in the region where Range 2 PC2 have negative values. The red spectrum shows the main dust band between 850 and $1200 \mathrm{~cm}^{-1}$ and the minor dust band between 300 and $550 \mathrm{~cm}^{-1}$. Red spectra in the scatter plot have Range 2 PC2 positive values, as well Range 1 PC2 values. Violet spectra are spread on dark regions of the albedo map, while yellow and green spectra are well grouped on the map and show the olivine features. We can note how the red and blue spectra are distributed on the typical three pixels TES narrow strips, pointing out their dependence with $L_{\mathrm{s}}$ and atmospheric conditions.

This method has been applied also to the Sinus Meridiani cube, where the first and largest grey hematite area has been identified by the TES team (Christensen et al. 2000b; Christensen et al. 2001a; Lane et al. 2001). Figure 2a shows Range 1 - Range 2 PC2 scatter plot of the cube with a spatial coverage of $5^{\circ} \mathrm{S}<$ Lat $<5^{\circ} \mathrm{N}$ and $0^{\circ} \mathrm{W}<$ Lon $<10^{\circ} \mathrm{W}$ and the location of spectral classes are reported on the albedo map in Fig. 2b. The mean spectra are shown in Fig. 2c. Also in this case we can note how (1) the spectra with dust are distributed in the region with Range 2 PC2 positive, (2) spectra with ice have Range 2 PC2 negative, (3) spectra with surface features have near zero Range 2 PC2 and negative Range 1 PC2. Dark violet, violet, yellow and green spectra show different percentage of dust (higher in the dark violet spectrum and minor in the green one) and the same hematite signatures. They are spatially grouped on the albedo map and seem to be aligned on a straight line on the scatter plot. The red spectrum has a high percentage of dust but does not have the surface hematite features, in fact these spectra are distributed out of hematite zone on the albedo map.

The Range 1 - Range 2 PC2 scatter plot can be physically interpreted as mappable features and spectral slope changes: dust spectra with typical negative slopes in the $800-1100 \mathrm{~cm}^{-1}$ range have positive Range 2 PC2 values and spectra with positive slopes in the $800-1100 \mathrm{~cm}^{-1}$ range, related to presence of ice, have negative Range 2 PC2 values. Range 1 PC2 values are related to the albedo as well: dark region spectra have Range 1 PC2 negative and bright region spectra have Range 1 PC2 values near zero. This gives the scatter plot diagrams the typical form of a boomerang. The length of the arms depends on the dust and ice content in the atmosphere. High spectral variance related to surface features has Range 1 PC2 values strongly negative and Range 2 PC2 values near zero.

Although this method allows us to map the distribution of olivine and hematite in these cubes, it does not work very well in the case of spectral features related to smaller regions, as in the case of the outcrop of hematite in Valles Marineris (Christensen et al. 2001a). For this reason we have introduced the CHISQR parameter.

CHISQR parameter. This is the CHI-square error in the linear fit of TES spectra between 300 and $550 \mathrm{~cm}^{-1}$. We have chosen this parameter because in this spectral range the effect of the dust is to introduce a negative slope to the spectrum, if it is present in the atmosphere, or to smooth spectral features, if it is deposited on the surface. In both these cases the spectral range considered is well fitted by a straight line with a more or less negative slope, respectively, and CHISQR values will be small see (Fig. 3). On the other hand, in the case of spectra with a high spectral variance due to superficial mineral absorptions, the linear fit will not be good, as well as in the case of spectra with high ice content, having absorptions before $300 \mathrm{~cm}^{-1}$ and after $450 \mathrm{~cm}^{-1}$, and CHISQR values will be high. In Fig. 3 we have reported mean spectra from the cube centered on Candor Chasma and Ophir regions where smaller outcrops of hematite have been identified (Christensen et al. 2001a). The first spectrum from the top presents higher content of dust than the other ones. The absorption between 800 and $1200 \mathrm{~cm}^{-1}$ is deeper and the shape of the spectrum between 300 and $550 \mathrm{~cm}^{-1}$ is characterized by a smooth negative slope that is well reproduced by the linear fit (small CHISQR value). The second spectrum presents a minor dust content. The band absorption between 800 and $1200 \mathrm{~cm}^{-1}$ is lower, as well as the negative slope between 300 and $550 \mathrm{~cm}^{-1}$. But again the spectrum in the last range is very smooth and the linear fit is good (small CHISQR value). The third spectrum has a high content of water ice (deep absorption before $1000 \mathrm{~cm}^{-1}$ and minor absorptions before $300 \mathrm{~cm}^{-1}$ and after $450 \mathrm{~cm}^{-1}$ ). The shape of the spectrum is not well reproduced by a straight line and the CHISQR value is then higher than in the previous cases. The last spectrum is an average of the TES pixels reported in Fig. 5. The hematite spectral signatures are distinctly visible, and because of the presence of band absorptions the spectrum is not smooth between 300 and $550 \mathrm{~cm}^{-1}$ and the CHISQR value is high. To distinguish between spectra with high values of the CHISQR parameter but with a different water ice content the PCA scatter plot can be used. In conclusion, the CHISQR allows us to identify spectra with high variance and the PCA allows us to discriminate within these spectra the ones that have ice and the ones having features likely related to surface. In Fig. 4 we have reported the Range 1 - Range 2 PC2 scatter plot for the Valles Marineris cube centered on Ophir and Candor Chasma, $10^{\circ} \mathrm{S}<$ Lat $<0^{\circ} \mathrm{S}$ and $70^{\circ} \mathrm{W}<$ Lon $<80^{\circ} \mathrm{W}$. We have marked with blue asterisks points with CHISQR $>0.002$ and with red asterisks the remaining points with high value for CHISQR related to hematite spectra. Blue points are localized in the ice spectral class, while red points are spread on the scatter plot. TES pixel positions of some red asterix points are reported on MOC image M0204452 (Malin et al. 2000) in Fig. 5, they are located on the same Ophir region reported by Christensen et al. (2001a).

The other reason why we have computed the CHISQR is because it is a more general parameter than the hematite index used by Christensen et al. (2001a, 2000b) to identify TES pixels with hematite. In fact, CHISQR is not defined by using some particular TES spectral channels but is related to the entire $300-550 \mathrm{~cm}^{-1}$ spectral range, so that, by means of CHISQR, we have been able to discriminate between both spectra with hematite and olivine and spectra with features due to other minerals or mineral mixtures, shown in the next section. 


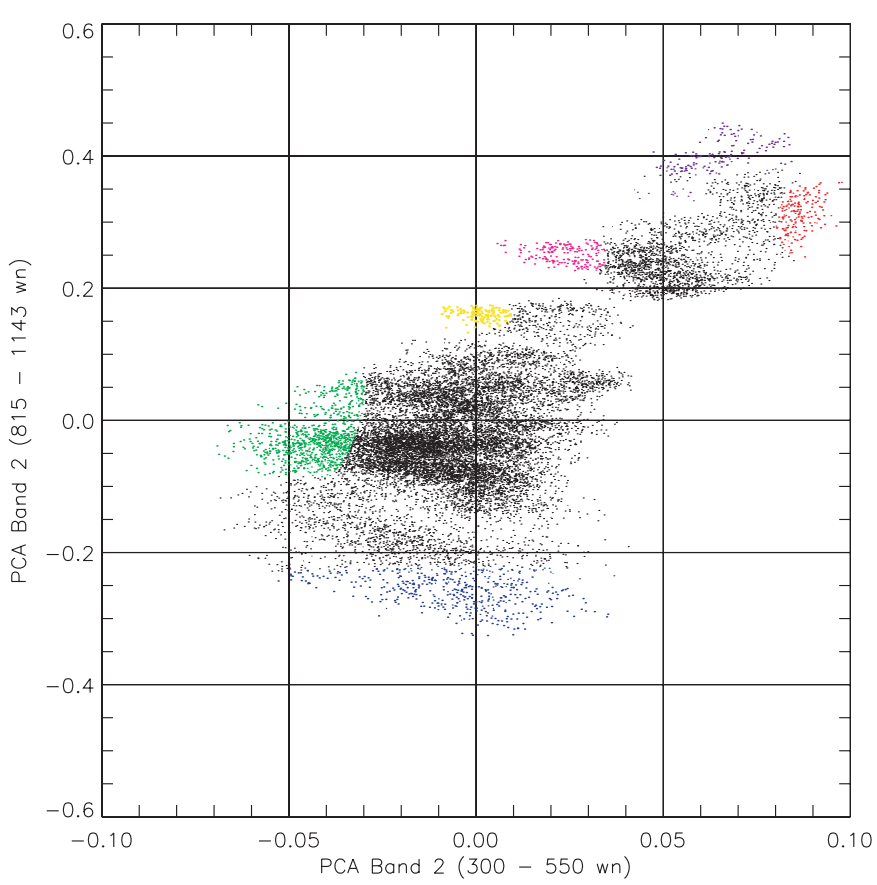

Fig. 2. a) PCA scatter plot of spectra from Sinus Meridiani region cube.

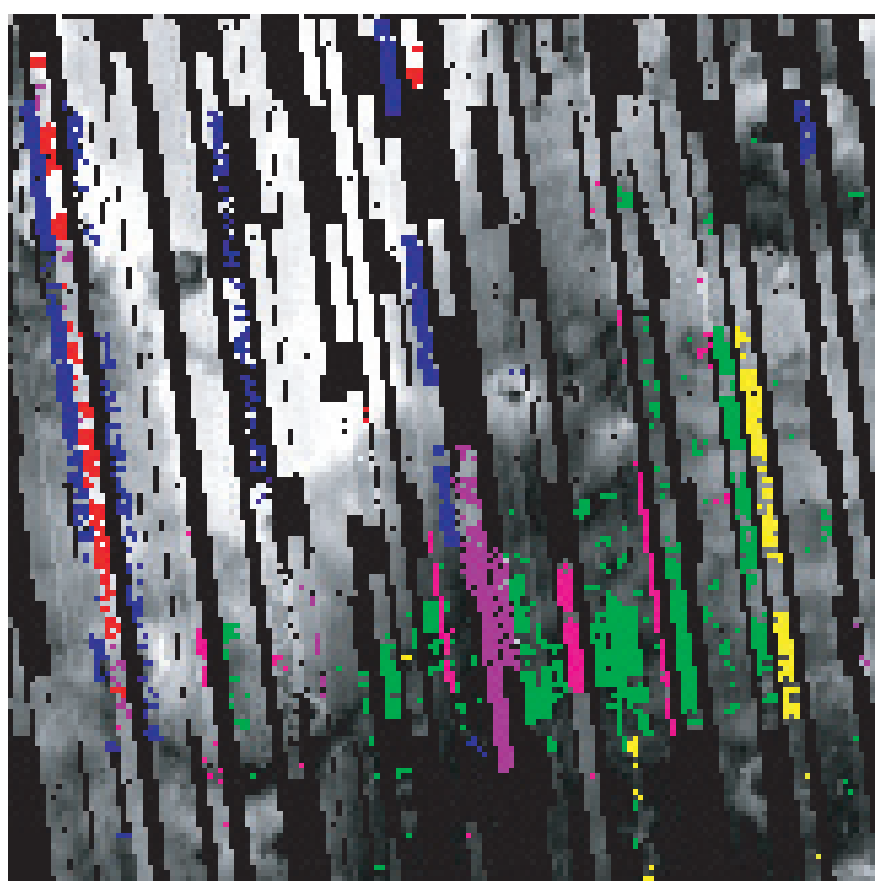

Fig. 2. b) Location on the albedo map of the spectral classes. Spatial coverage $5^{\circ} \mathrm{S}<$ Lat $<5^{\circ} \mathrm{N}$ and $0^{\circ} \mathrm{W}<$ Lon $<10^{\circ} \mathrm{W}$.

\section{TES spectra}

Spectra with high values for the CHISQR parameter and low water ice content have been found in northeastern Arabia Terra (Lon $\sim 294^{\circ} \mathrm{W}$, Lat $\left.\sim 27^{\circ} \mathrm{N}\right)$. In Fig. 6 we have reported a Viking image including the Baldet crater with the superimposition of TES pixels showing significant variance in the spectral range between 300 and $500 \mathrm{~cm}^{-1}$. White boxes in Fig. 6 show typical signatures of hematite. The mean spectrum is labelled

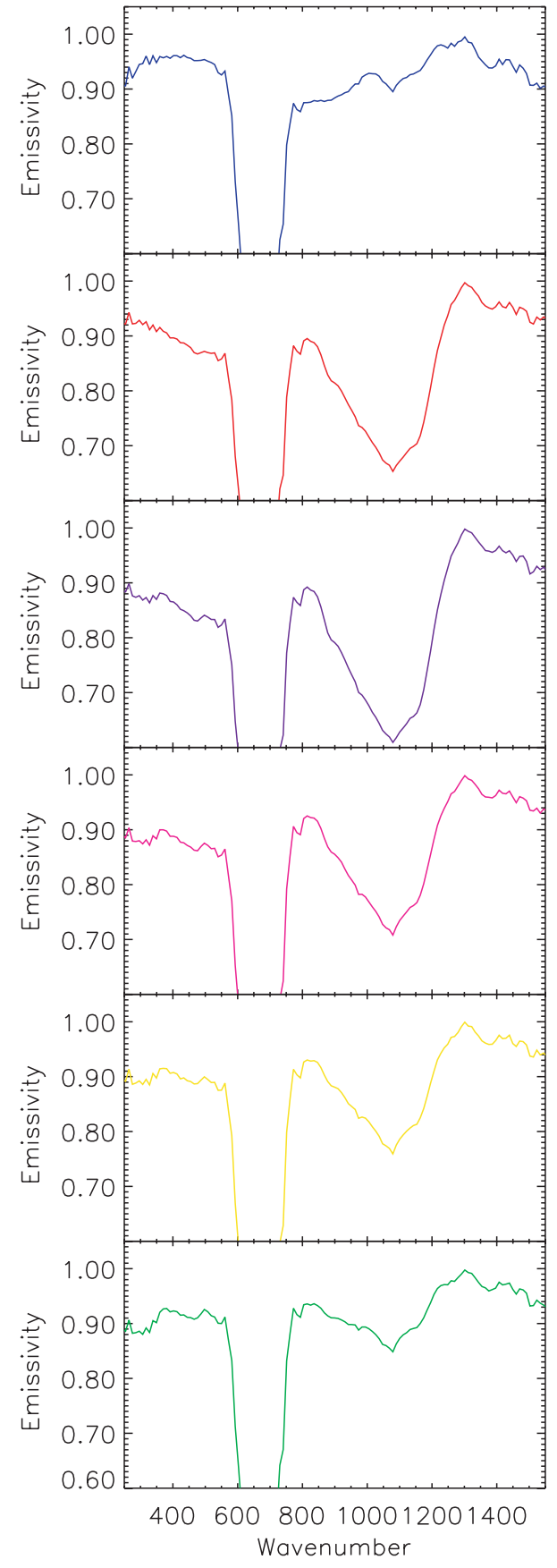

Fig. 2. c) Mean spectra of the classes reported in Fig. 2a. Blue and red spectra have water ice and dust bands. The other spectra show a different percentage of dust and the surface hematite features in the $300-500 \mathrm{~cm}^{-1}$ spectral range.

in Fig. 7a as Sp A. The mean spectrum of the Sinus Meridiani hematite spectra is indicated as SM and has been plotted for comparison. The hematite index value for the SM mean spectrum is 1.028 and for the $\mathrm{Sp} \mathrm{A}$ spectrum is 1.015. The detection limit for the hematite band indicated in Christensen et al. (2001a) is 1.018, and the hematite index value found for the vast majority of TES spectra is $\sim 1.010$, with a variation of \pm 0.002 consistent with instrumental noise. As reported in Christensen et al. (2000a) high values of the hematite index can be found also for spectra with significant water ice 

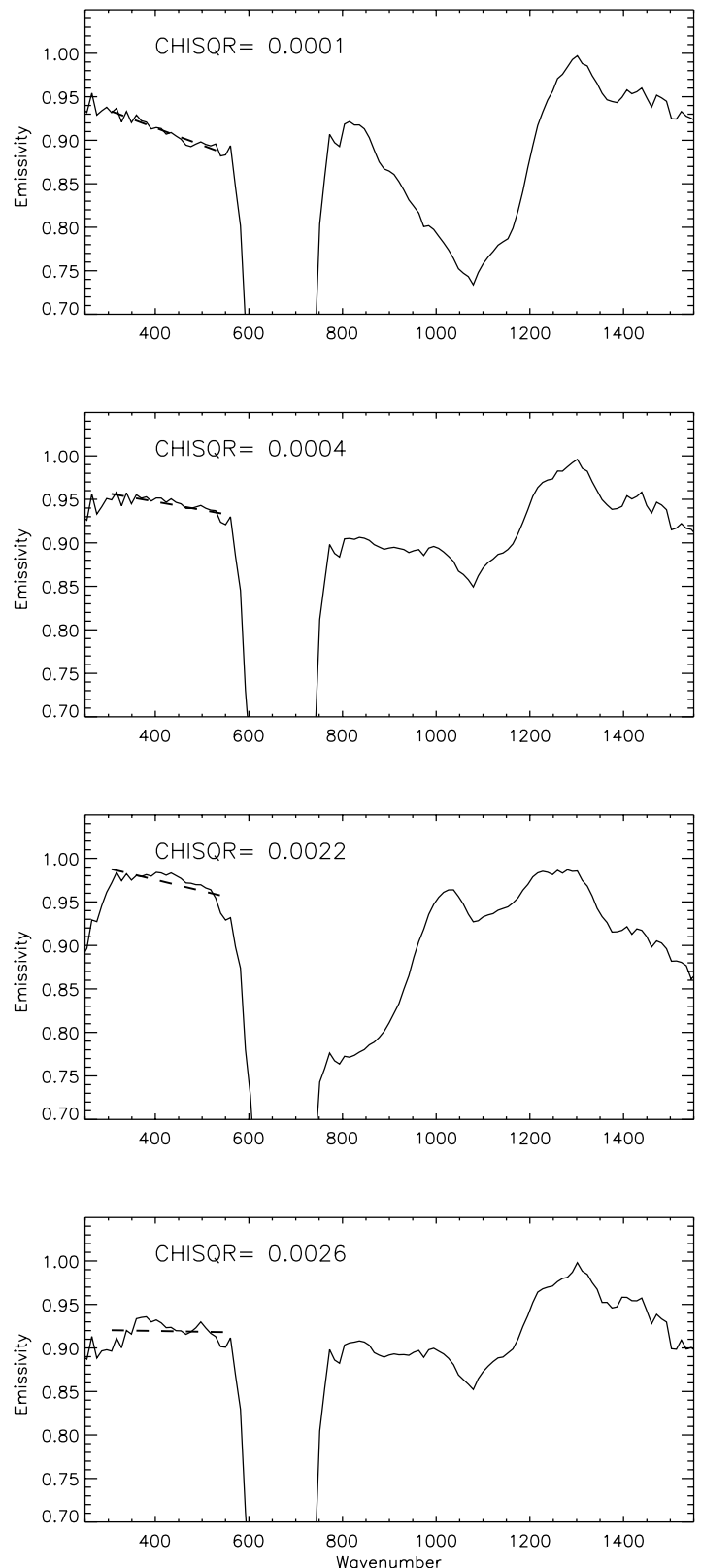

Fig. 3. Mean spectra selected from the Candor Chasma and Ophir cube. We have selected spectra with high dust content, spectra with low dust content, spectra with high water ice content and spectra with the signatures of hematite. For each one of these spectral classes we have computed the linear fit between 300 and $550 \mathrm{~cm}^{-1}$ and the CHISQR values. The fit is reported with a dashed line. From the plots it is evident how there is a good correspondence between the linear fit and the first tow spectra from the top (low values for CHISQR parameter), while in the case of spectral classes with water ice and surface signatures the spectral shape in the range selected is not so smooth (high values for the CHISQR parameter).

opacity. This implies that the mean value of the hematite index increases if any selection is done in the TES spectra and if data with a high content of water ice are included. Choosing spectra close to the area mapped in Fig. 6 and with low ice content and no hematite signatures, we have found a hematite index value for TES data of $\sim 1.004$, and the value of 1.015

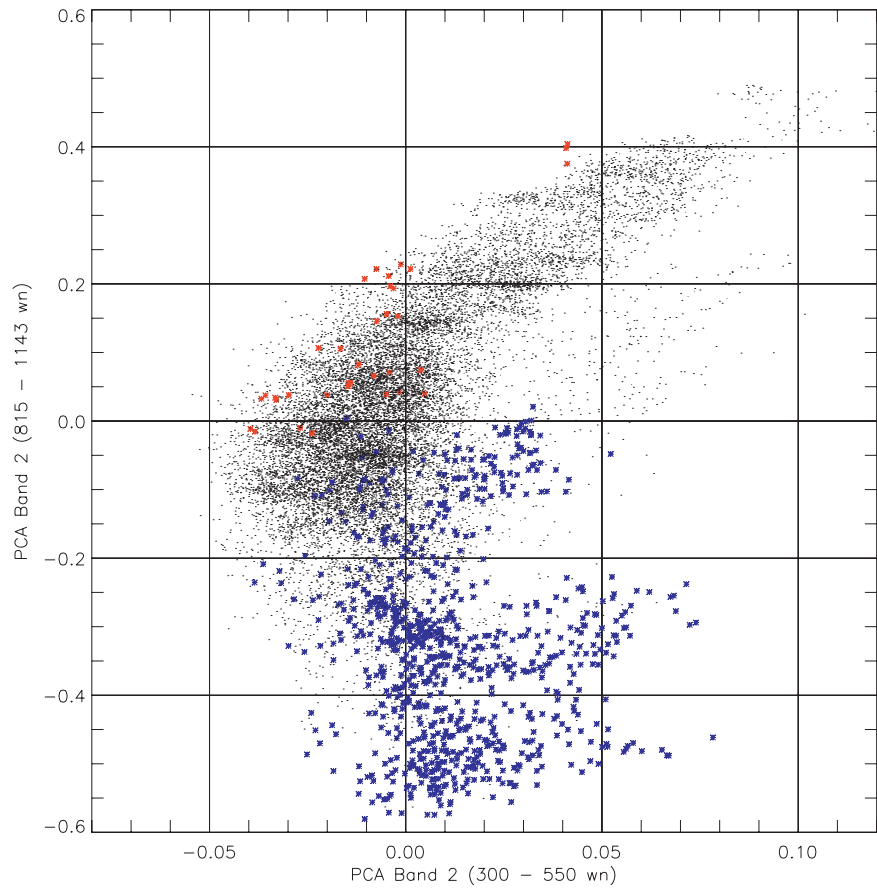

Fig. 4. PCA scatter plot for Candor Chasma and Ophir cube. Spectra with CHISQR $>2$ have been marked with a blue asterisk. Spectra with hematite features have been marked with a red asterisk.

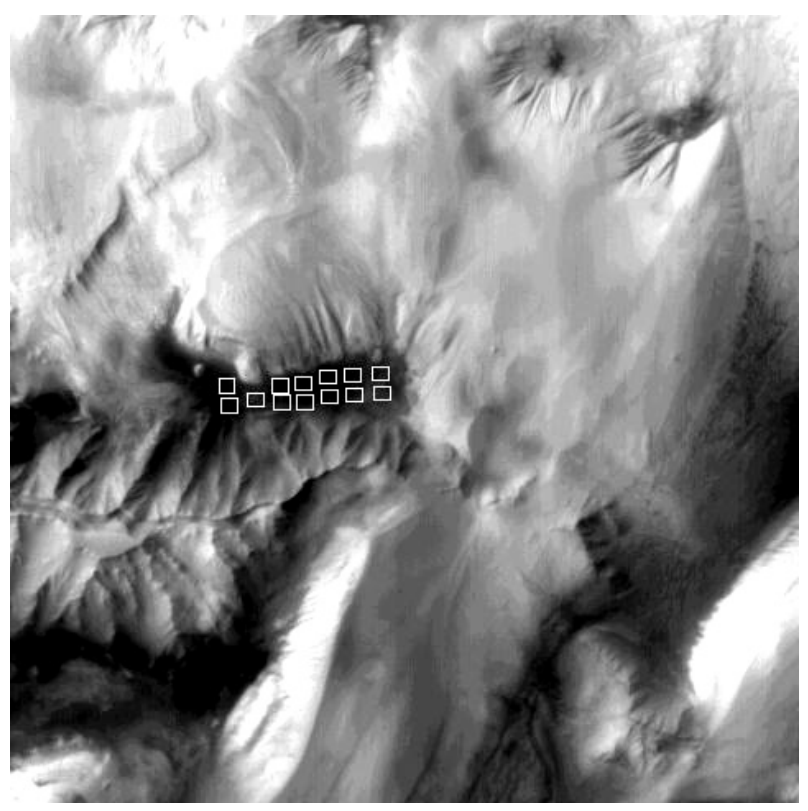

Fig. 5. MOC image M0204452 (Malin et al. 2000) centered at $72.8^{\circ} \mathrm{W}$ and $4.70^{\circ} \mathrm{S}$ on the Ophir region with the location of TES spectra showing the hematite features. For the coordinate errors on MOC images, the position of TES hematite spectra on the M0204452 image has been obtained combining the spectra latitude and longitude values with the TES albedo channel values.

could be considered acceptable. The different band contrast at $\sim 300 \mathrm{~cm}^{-1}$ of $\mathrm{Sp}$ A compared with SM spectrum could be due to a different hematite abundance and the different shape of the maximum at $\sim 500 \mathrm{~cm}^{-1}$ could be due to the presence of other mixed materials (Morris et al. 2001) or of a surface dust coating that masks their real abundance (Gaff et al. 2001; 


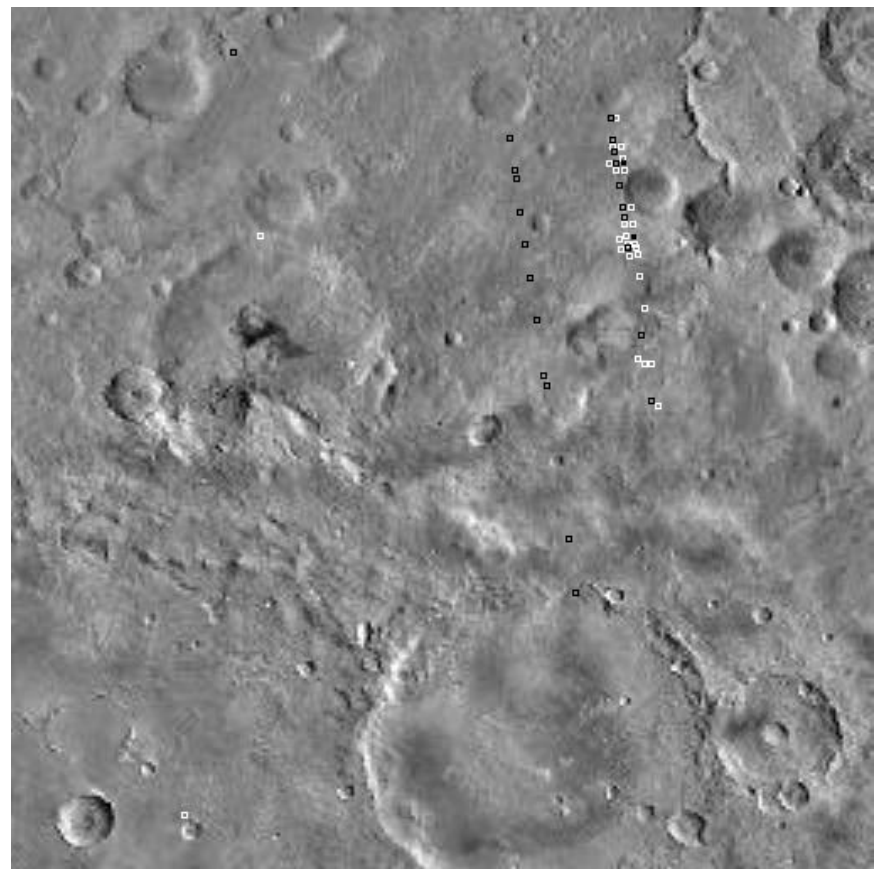

Fig. 6. Viking image with locations of the TES pixels showing interesting features between $300 \mathrm{~cm}^{-1}$ and $500 \mathrm{~cm}^{-1}$. The bigger crater on the bottom is Baldet crater. On the top right area of the image the final portion of Huo Hsing Vallis is visible. White empty pixels have typical absorptions of hematite spectra, black filled pixels show a band absorption at $360 \mathrm{~cm}^{-1}$ and black empty pixels have a band absorption at $390 \mathrm{~cm}^{-1}$.

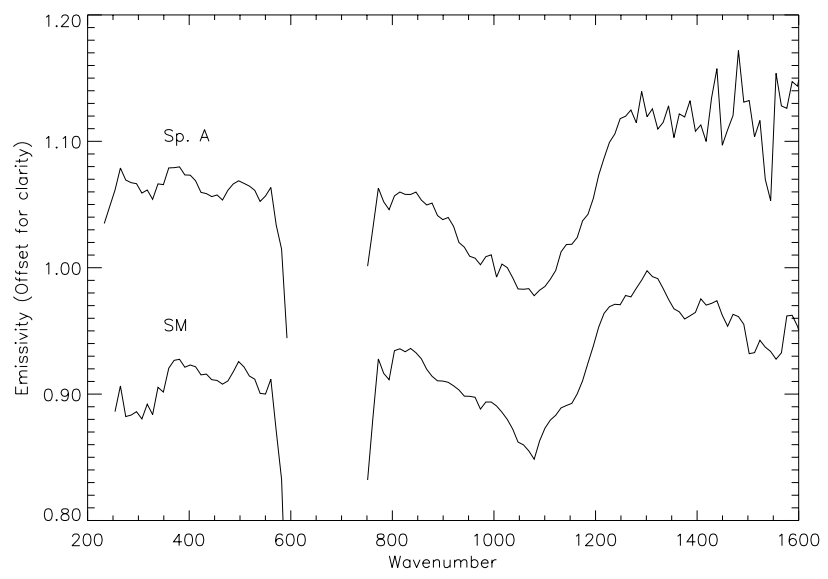

Fig. 7. a) The first spectrum on the bottom is the hematite mean spectrum from Sinum Meridiani. The Sp A spectrum is the average of white empty TES pixels in Fig. 6.

Crisp \& Bartholomew 1992). The average of the two spectra with black filled pixels is reported in Fig. $7 \mathrm{~b}$ as Sp B. A band absorption centered at $\sim 360 \mathrm{~cm}^{-1}$ is clearly visible and is perhaps due to magnetite. The band depth, in percent, of the TES $360 \mathrm{~cm}^{-1}$ feature is 2.1 , while the band detection limit on the same spectral region computed by Kirkland et al. (2001) for TES is of the order of $\sim 1.4$, assuming a confidence factor of 2 for the peak to peak noise and a target band full width at the half of the maximum of the band depth of $30 \mathrm{~cm}^{-1}$. In Fig. 7c we have reported the mean spectrum of the dark empty pixels as Sp C. A band centered at $\sim 390 \mathrm{~cm}^{-1}$ is visible as well as

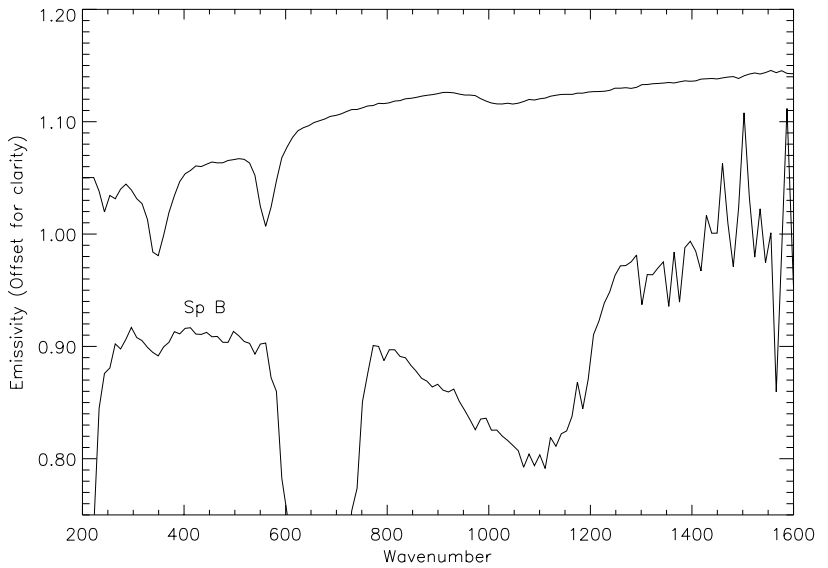

Fig. 7. b) The first spectrum on the bottom is the mean spectrum of the two black filled pixels in Fig. 6 having a band absorption centered at $\sim 360 \mathrm{~cm}^{-1}$. The ASU Thermal Emission Spectral Library magnetite sample (Christensen et al. 2000c) shows an absorption at the same wavenumber.

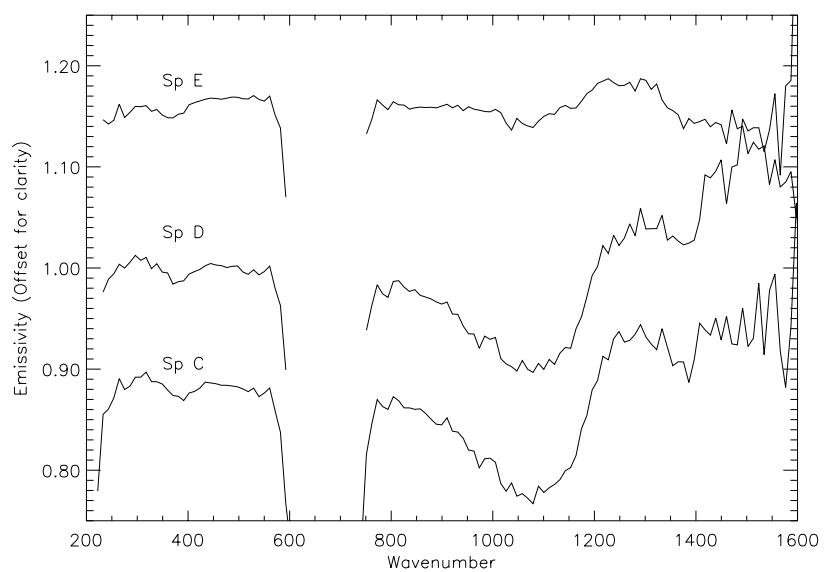

Fig. 7. c) Spectrum $\mathrm{Sp} \mathrm{C}$ is the average of the black empty pixels in Fig. 6 showing a band absorption centered at $\sim 390 \mathrm{~cm}^{-1}$. Spectra with similar features have been identified spread over areas near Syrtis Major, Sp D, and on Sinai Planum, Sp E.

in Sp D and Sp E, identified in different areas, respectively near Syrtis Major and on Sinai Planum. The band depth for Sp C is 1.9 , for Sp D is 2.3 and for $\mathrm{Sp} \mathrm{E}$ is 1.5 . The majority of the spectra mapped in Fig. 6 come from the same TES orbit and are located near Huo Hsing Vallis. This region presents a heavily cratered terrain, characterized by a mantling deposit of horizontally layered material subsequently subjected to erosion (Moore 1990). Erosion of this extent suggests that the material is easily broken down into transportable elements. Speculative origins for the deposit include formation as differently welded pyroclastic tuff or a compacted aeolian dust deposit. The hypothesis that the deposit could have an underformed, horizontal, massive, water-laid sedimentary origin has been discarded by Moore (1990) due to the lack of compelling fluvial or lacustrine geomorphic features on the Mars highlands. The understanding of all minerals responsible for the observed bands in the $300-550 \mathrm{~cm}^{-1}$ TES spectral range could give us new clues about the past history and formation of this region of the planet. 


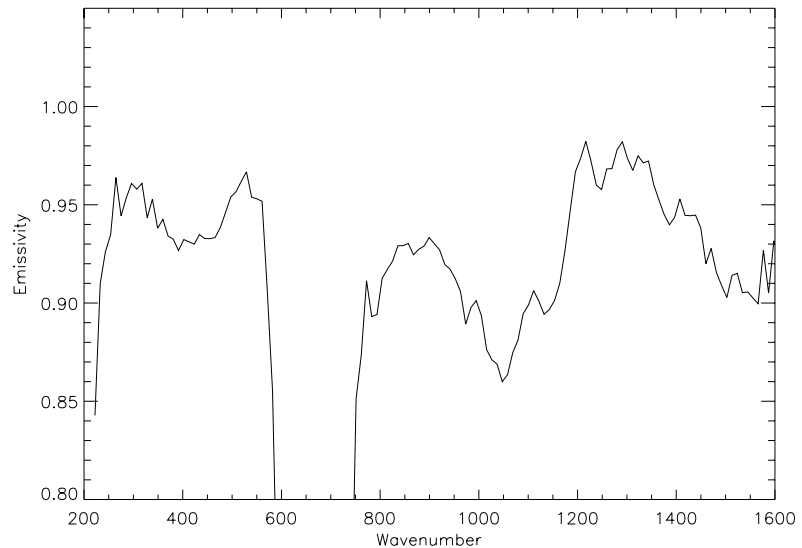

Fig. 8. Mean TES spectrum having a broad absorption between 300 and $500 \mathrm{~cm}^{-1}$.

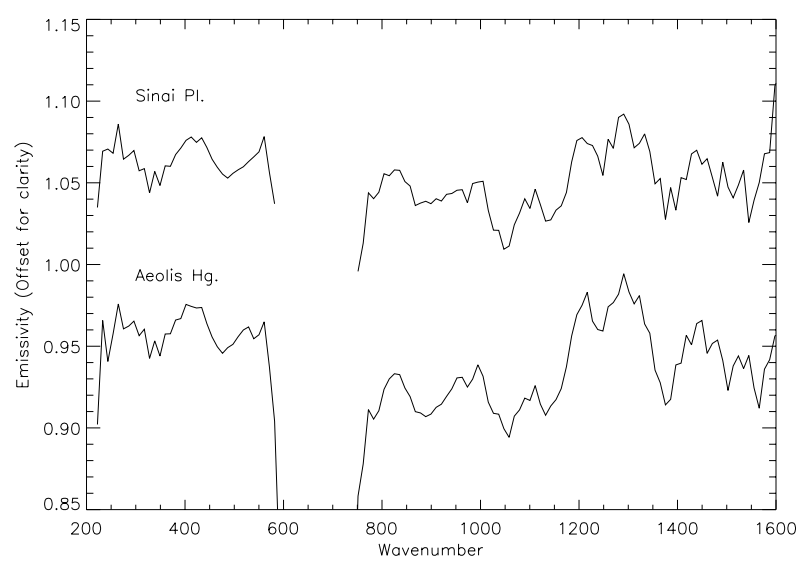

Fig. 9. The Sinai Pl. spectrum is the average of six adjacent TES pixels from Sinai Planum, the Aeolis Hg. spectrum is the average of five adjacent pixels collected on a region near Aeolis Highland. These two spectra acquired at different longitudes show same signatures at $\sim 340$, $\sim 410 \mathrm{~cm}^{-1}$ and $\sim 490 \mathrm{~cm}^{-1}$.

In Fig. 8 we have reported the mean of fifteen single TES spectra coming from adjacent sensors and acquired on areas centered at $215.3^{\circ} \mathrm{W}$ and $13.6^{\circ} \mathrm{N}, 222.6^{\circ} \mathrm{W}$ and $0.1^{\circ} \mathrm{N}$, $238.6^{\circ} \mathrm{W}$ and $18.0^{\circ} \mathrm{S}$. A broad band absorption centered at $\sim 400 \mathrm{~cm}^{-1}$ is clearly visible.

In Fig. 9 the average of five TES spectra from Aeolis Highland (Aeolis Hg.) and six TES spectra from Sinai Planum (Sinai Pl.) are reported. Although they have been collected at different longitudes, they show similar signatures: absorptions at $\sim 340$ and $\sim 490 \mathrm{~cm}^{-1}$ and a maximum at $\sim 410 \mathrm{~cm}^{-1}$. We have not computed the band depth for these spectra since it is not clear where to assume the continuum lies. In fact the band at $490 \mathrm{~cm}^{-1}$ is cut on the right by $\mathrm{CO}_{2}$ absorptions and the band at $340 \mathrm{~cm}^{-1}$ is on the left border of the TES spectral range.

As the principal purpose of this study is the description of the spectral statistical analysis applicable for mapping the surface of Mars, a best understanding of the new spectral features observed between $300 \mathrm{~cm}^{-1}$ and $500 \mathrm{~cm}^{-1}$ and shown in this section is reserved for future works, as is as the discussion about other absorptions present between $800 \mathrm{~cm}^{-1}$ and $1300 \mathrm{~cm}^{-1}$. Only the library spectrum of magnetite has been reported due to the evident similarity between the laboratory and the TES spectra. Magnetite has been suggested as a component of magnetic material found in Martian soil (Hargraves et al. 1979; Pollack et al. 1977) and has been identified in some Martian meteorites (Scott 1999).

Although the number of spectra reported in this study is small compared to the huge amount of data collected by TES, they show between $300 \mathrm{~cm}^{-1}$ and $500 \mathrm{~cm}^{-1}$ features that could give new information about Martian surface properties.

We want to stress here the fact that, except for rare exceptions, the Martian surface does not show strong spectral variations at spatial scales $\geq 10 \mathrm{~km}$ in the $300-500 \mathrm{~cm}^{-1}$ spectral range. This suggests that only spectral analysis at higher spatial resolution can give new clues on Martian mineralogy. Clays and carbonates are expected to exist on Mars if water was abundant in the past (Pollack et al. 1987; Catling 1999). A failure in detection of these minerals could be due to the fact that they occur in small patches (areal coverage $\leq 10 \mathrm{~km}^{2}$ ) on the Martian surface and in low concentrations. This was the main reason why we developed a method to identify single TES spectra (spatial footprint $\sim 3 \mathrm{~km} \times 3 \mathrm{~km}$ ).

\section{Conclusions}

There are two principal conclusions that we can draw from this study. The first one is that the combined use of a Range $1-$ Range 2 PC2 scatter plot with the CHISQR parameter allows us to identify single TES data with high spectral variance in the $300-500 \mathrm{~cm}^{-1}$ range attributable to superficial components. The Range 1-Range 2 PC2 scatter plot characterizes in each cube spectral classes having similar dust content, similar ice content or similar superficial features. If data with interesting $300-500 \mathrm{~cm}^{-1}$ spectral features are limited and spread on the Martian surface, then the use of the CHISQR parameter is necessary. In this way we have been able to identify hematite outcrops in Valles Marineris, already studied by the TES team, and new unique spectra in a region between northeastern Arabia Terra and Syrtis Major, in Sinai Planum, and spread over a region between west Elysium Planitia and Terra Cimmeria. The fact that these bands have characteristics distinct from those observed in the atmospheric bands lets us suppose that they can be caused by the surface components, as in the case of hematite.

The second conclusion is that on the Martian surface there are spots with a dimension of $\sim 3-10 \mathrm{~km}$ whose spectra show absorption bands between 300 and $500 \mathrm{~cm}^{-1}$ never observed in previous studies. The signatures they show recur at some specific wavenumbers, so that these bands could be related to some specific mineralogical typologies. If these observed spots are outcrops of the same dimension of the order of $\sim 3-10 \mathrm{~km}$, or if they are larger but spectrally obscured by a surface dust coating is not still clear. The interpretation of these spectra in terms of possible mineralogy will be the object of a future study.

Acknowledgements. Authors wish to thank Mrs S. Zampieri and Dr. L. Di Fino for the help in the manuscript preparation and Dr Scott Nowicki for the careful review of the paper. Funding were provided by ASI grants. 


\section{References}

Arvidson, R. E., Guinness, E. A., \& Zent, A. P. 1982, J. Geophys. Res., 87, 10149

Bell, J. F. III, Lucey, P. G., \& McCord, T. B. 1992, Experimental Astronomy, 2, 287

Catling, D. C. 1999, J. Geophys. Res., 104, 16453

Christensen, P. R., Anderson, D. L., Stillman, C. C., et al. 1992, J. Geophys. Res., 97, 7719

Christensen, P. R., \& Harrison, S. T. 1993, J. Geophys. Res., 98 (B11), 19819

Christensen, P. R. 1997, J. Geophys. Res., 103, 1733

Christensen, P. R. 1999, Calibration Report for the Thermal Emission Spectrometer (TES) for the Mars Global Surveyor Mission, Mars Global Surveyor Project, Jet Propul. Lab., Pasadena, Calif.

Christensen, P. R., Bandfield, J. L., Smith, M. D., et al. 2000a, J. Geophys. Res., 105, 9609

Christensen, P. R., Bandfield, J. L., Clark, R. N., et al. 2000b, J. Geophys. Res., 105, 9623

Christensen, P.R., Bandfield, J. L., Hamilton, V. E., et al. 2000, J. Geophys. Res., 105, 9735

Christensen, P. R., Morris, R. V., Lane, M. D., et al. 2001a, J. Geophys. Res., 106, 22873

Christensen, P. R., Bandfield, J. L., Hamilton, V. E., et al. 2001b, J. Geophys. Res., 106, 23823

Crisp, J., \& Bartholomew, M. J. 1992, J. Geophys. Res., 97, 14691

Davis, J. C. 1986, in Statistics and Data Analysis in Geology (New York: John Wiley and Sons Pubs.), 527

Graff, T. G., Morris, R. V., \& Christensen, P. R. 2001, Effects of Palagonitic Dust Coatings on Thermal Emission Spectra of Rocks and Minerals: Implications for Mineralogical characterization of the Martian Surface by MGS-TES, Lunar and Planet. Sci [CDROM], XXXII, Abstract 1899

Grassi, D., \& Formisano, V. 2000, Planet. Space Sci., 48, 577

Hamilton, V. E., Christensen, P. R., \& McSween, H. Y. 2001, Spectral variations in MGS TES Data of Nili Fossae: A possible source region for SNC Meteorites on Mars?, Lunar and Planet. Sci. [CDROM], XXXII, Abstract 2184

Hargraves, R. B., Collinson, D. W., Arvidson, R. E., \& Cates, P. M. 1979, J. Geophys. Res., 84, 8379
Hoefen, T. M., \& Clark, R. N. 2001, Compositional Variability of Martian Olivines Using Mars Global Surveyor Thermal Emission Spectra, Lunar and Planet. Sci [CD-ROM], XXXII, Abstract 2049

Hoefen, T. M., Clark, R. N., Pearl, J. C., et al. 2000, Unique Spectral features in Mars Global Surveyor Thermal Emission Spectrometer: Implication for Surface Mineralogy in Nili Fossae, American Astronomical Society, DPS meeting, 32, 62.03

Huguenin, R. L. 1987, Icarus, 10, 162

Kirkland, L. E., Herr, K. C., Salisbury, J. W., et al. 2001, Detecting Minerals on Mars using TES, THEMIS, and min-TES, Workshop on the Martian Highlands and Mojave Desert Analogs (2001), Abstract 4025

Lane, M. D., Hartmann, W. K., Berman, D. C., et al. 2001, Update on studies of Martian hematite-rich areas, Lunar and Planet. Sci. [CD-ROM], XXXII, Abstract 1984

Maguire, W. C. 1977, Icarus, 32, 85

Malin, M. C., Edgett, K. S., Davis, S. D., et al. 2000, M0204452, Malin Space Science Systems Mars Orbiter Camera Image Gallery (http://www.msss.com/moc_gallery/), 22 May 2000

Moore, J. M. 1990, J. Geophys. Res., 95, 14279

Morris, R. V., Lane, M. D., Mertzman, S., Shelfer, T. D., \& Christensen, P. R. 2001, Chemical and Mineralogical Purity of Sinus Meridiani Hematite, Lunar and Planet. Sci [CD-ROM], XXXI, Abstract 1618

Pollack, J. B., Colburn, D., Kahn, R., et al. 1977,J. Geophys. Res., 82, 4479

Pollack, J. B., Kasting, J. F., Richardson, S. M., et al. 1987, Icarus, 71, 203-224

Ruff, S. W., Christensen, P. R., Barbera, P. W., et al. 1997, J. Geophys. Res., 102, 14899

Salisbury, J. W., \& Walker, L. W. 1989, J. Geophys. Res., 94, 9192

Salisbury, J. W., Walter, L. S., Vergo, N., et al. 1991, in Infrared (2.1-25 $\mu \mathrm{m}$ ) Spectra of Minerals, 267 pp. (Baltimore, Md.: Johns Hopkins Univ. Press)

Scott, E. R. 1999, J. Geophys. Res., 104, 3803

Siegal, B. S., \& Gillespie, A. R. 1980, Remote Sensing in Geology, (New York: John Wiley and Sons Pubs.), 200 pp.

Smith, M. D., Christensen, P. R., \& Bandfield, J. L. 1999, J. Geophys. Res., 105, 9589 